\title{
Efficient power component identification with long short-term memory and deep neural network
}

\author{
Jingsheng Lei ${ }^{1}$, Wenbin Shi ${ }^{1,3^{*}}$ (D), Zhichao Lei ${ }^{2}$ and Fengyong $\mathrm{Li}^{1}$
}

\begin{abstract}
This paper tackles a recent challenge in patrol image processing on how to improve the identification accuracy for power component, especially for the scenarios including many interference objects. Our proposed method can fully use the patrol image information from live work, and it is thus different from traditional power component identification methods. Firstly, we use long short-term memory networks to synthesize the context information in a convolutional neural network. Then, we constructed the Mask LSTM-CNN model by combining the existing Mask $\mathrm{R}-\mathrm{CNN}$ method and the context information. Further, by extracting the specific features belonging to the power components, we design an optimization algorithm to optimize the parameters of Mask LSTM-CNN model. Our solution is competitive in the sense that the power component is still identified accurately even if the patrol images contain much interference information. Extensive experiments show that the proposed scheme can improve the accuracy of component recognition and has an excellent anti-interference ability. Comparing with the existing R-FCN model and Faster R-CNN model, the proposed method demonstrates a significantly superior detection performance, and the average recognition accuracy is improved from 8 to $11 \%$.
\end{abstract}

Keywords: Power component identification, Long short-term memory, Convolutional neural network, Anti-interference, Live work inspection

\section{Introduction}

With the rapid development of artificial intelligence, live working robots that can perform automatic inspection have received extensive attention from major power grid corporations [1]. For power systems, blackouts mean a drop in economic efficiency [3]. To maintain a good operating condition of the equipment, machine vision is added in a live working robot to obtain more information about the environment [4] and provide the ability of non-contact measurements. This ability does not pose any danger to workers and thus improve the safety of the system [2]. Also, machine vision is also able to replace the long-term work of the human eye so that the continuous monitoring and identification can be achieved successfully.

*Correspondence: 18817931275@163.com

${ }^{1}$ College of Computer Science and Technology, Shanghai University of Electric Power, Shanghai, 200090 People's Republic of China

${ }^{3}$ College of Automation Engineering, Shanghai University of Electric Power,

Shanghai, 200090 People's Republic of China

Full list of author information is available at the end of the article
Different from the traditional identification of power components [5], the images from live working robots have complex backgrounds, high density of parts, and high timeliness requirements and contain many interference objects. In this sense, traditional power component identification cannot be applied well to the patrol images from live working robots because they mainly use manually designed features and segmentation algorithm, where classical features include SIFT (scale-invariant feature transform) [6], edge detector [7], and HOG (histogram of oriented gradients) [8], while the segmentation algorithms are mainly based on peripheral contour skeleton [9] and adaptive threshold [10]. However, applying these methods to automatic detection is not practical due to the following drawbacks: (1) they are often based on specific categories in the design principle so that their accuracy is lower and the scalability is not stronger. and (2) these methods always have a loose structure and lack comprehensive utilization of low-level features to achieve the goal of optimal global identification. 
Compared with the traditional method, Ren et al. proposed a new approach, named by Faster-RCNN (faster region-based convolutional neural network) [11]. Regarding structure, Faster-RCNN has integrated feature extraction, proposal extraction, bounding box regression (rectangular refine), and classification into a network. It leads to a significant improvement in overall performance and detection speed. R-FCN is another target detection structure proposed by [12]. It modified the previous Faster-RCNN structure by moving the convolutions to the front of the ROI layer. R-FCN used a position-sensitive feature map to evaluate the probability of each category and was thus more accurate in positioning. Although the detection rate was improved, R-FCN cannot recognize the specific contour of the target. Due to this drawback, R-FCN has a limited range of application. Mask R-CNN was proposed by K. He [13], a researcher of Facebook AI, in 2017. This method expands the object detection technology and achieves pixel-level segmentation and contour segmentation of targets by using bilinear interpolation. Law method, proposed by [14], reduced the loss of space symmetry and has better recognition effect in scenes with a transparent background and foreground segmentation. However, it does not have sufficient adaptability to power component scenes with many interference factors and cannot fully exploit the image's associated information.

To improve the identification precision for power component, this paper proposes an efficient power component identification with long short-term memory and deep neural network. Based on Mask R-CNN, we design Mask LSTM-CNN model to integrate context features in the classification and regression layers through LSTM neural network [15]. Firstly, we use long short-term memory networks to synthesize the context information in a convolutional neural network. Then, we constructed Mask LSTM-CNN model by combining the existing Mask $\mathrm{R}-\mathrm{CNN}$ method and the context information. Further, by extracting the specific features belonging to the power components, we design an optimization algorithm to optimize the parameters of Mask LSTM-CNN model [16]. Extensive experiments show that the proposed scheme has an excellent anti-interference ability and verify that the power component is still identified accurately even if the patrol images contain much interference information. Comparing with the existing R-FCN model and Faster R-CNN model, the detection accuracy of proposed scheme has a significant improvement with a range from 8 to $11 \%$.

The rest of this paper is organized as follows. Section 2 presents several traditional power component identification schemes. In Section 3, we provide the details of the proposed approach and introduce the designing procedure of Mask LSTM-CNN. Subsequently, comprehensive experiments are performed to evaluate the performance of the proposed scheme. The experimental results and corresponding discussions are presented in Section 4, respectively. Finally, Section 5 concludes the paper.

\section{Related works}

\subsection{Power station identification based on Faster-RCNN method}

Following R-CNN [17] and Fast R-CNN [18], FasterRCNN was proposed by [11]. This method can identify region proposals by using a regional proposal network (RPN), which replaces previous methods such as Selective Search [19] and Edge Boxes [20]. RPN and the detection network share the convolutional characteristics of the whole map so that detection for a region can take less time [21]. The structure of the Faster-RCNN neural network is shown in Fig. 1.

RPN is a full convolutional-based network [22], and it can simultaneously predict the position of the target picture area and the target score (the probability value of the real target) of the input picture. Meanwhile, RPN is also an end-to-end network training method to generate high-quality regional proposal boxes for Fast R-CNN classification detection. With an optimization method, RPN and Fast $\mathrm{R}-\mathrm{CNN}$ can share convolution features during training. Combing these two models, an overall structure, named by "RPN+Fast R-CNN," is integrated. In this structure, RPN network is mainly used to generate high-quality proposal area boxes, while Fast R-CNN is used to learn high-quality proposed area features and classification.

Faster R-CNN designs the network RPN to extract candidate areas and replaces the selective search with

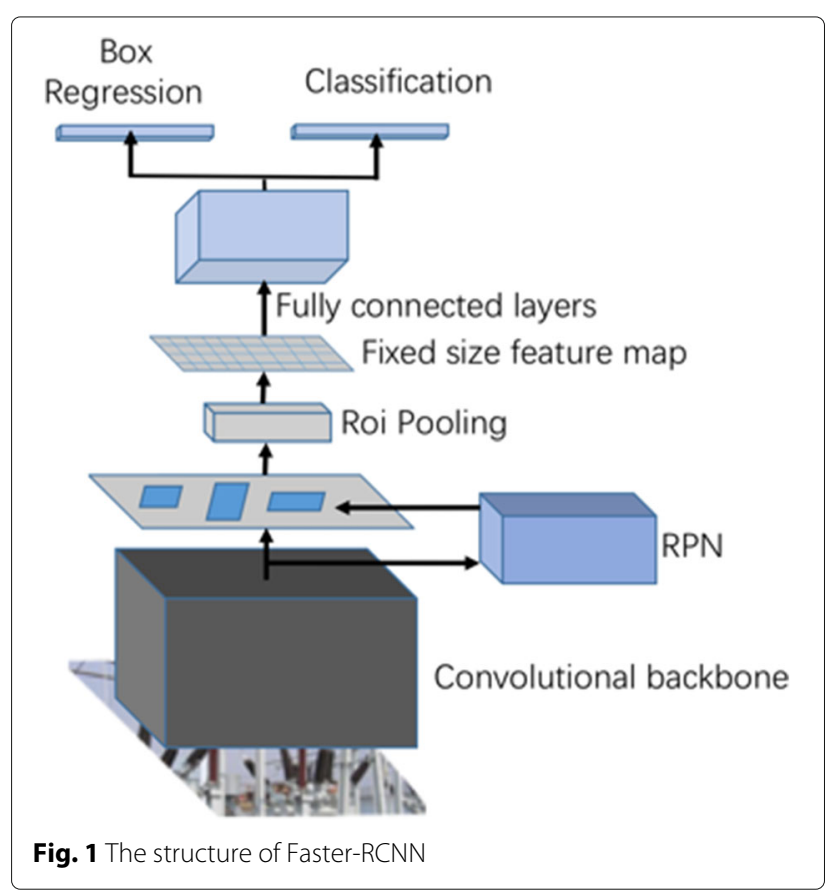




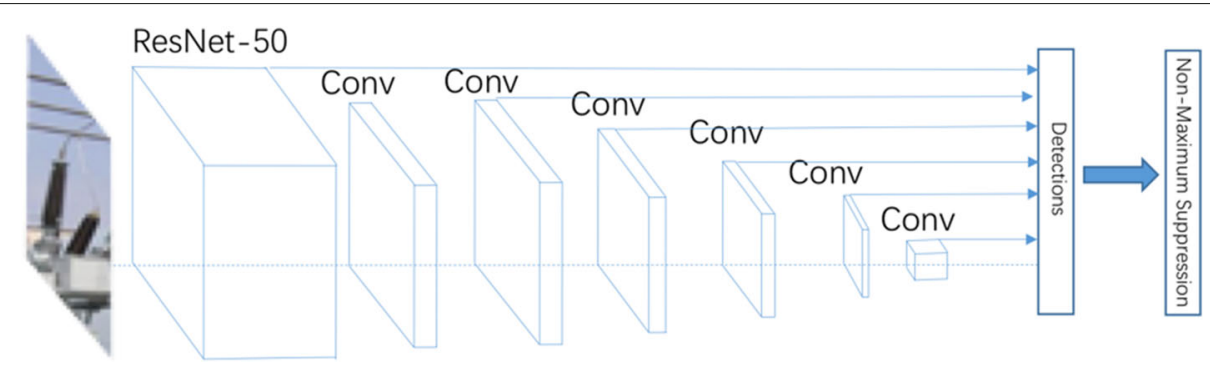

Fig. 2 The structure of R-FCN

lower efficiency. This process significantly improves the detection speed of the entire model. However, it can only determine the target's general location instead of the specific power component's position. Overall, this model has a low recognition rate when the power components are occluded. Thus, it cannot meet the on-site requirements for power component identification.

\subsection{Power station identification based on R-FCN method}

The target detection of the regional-based full convolutional network [22] is divided into two steps: positioning a target and then classifying the target to a specific category. First, R-FCN model uses a rudimentary convolutional network to generate a feature map. Then, the regional feature map is used to generate the feature map before and after the full map is constructed. The model determines the target's outline by searching and filtering [23] scene images through these feature maps. Finally, the classification framework recognizes the target.

Figure 2 demonstrates the structure of R-FCN model. The target image is passed through a basic convolutional network to generate feature maps and input these feature maps into a full-volume network to generate a score bank of position-sensitive score maps. The results of the basic convolutional network go through the RPN network to generate RoI. For a RoI of size $w \times h$ (obtained by the RPN network), the target frame is divided into $k \times k$ subareas, each subarea is of size $w \times h / k 2$. For anyone subarea $\operatorname{bin}(i, j), j \leq k-1$, define a location-sensitive pooling operation:

$$
r_{c}(i, j \mid \nabla)=\sum_{(x, y) \in \operatorname{bin}(i, j)} \frac{1}{n} z_{i, j, c}\left(x+x_{0}, y+y_{0} \mid \nabla\right)
$$

where $r_{c}(i, j \mid \nabla)$ is the pooled response of subarea $\operatorname{bin}(i, j)$ to $c$ categories and $z_{i, j, c}$ stands for a location-sensitive score map corresponding to subarea bin $(i, j) . x_{0}+y_{0}$ represents the coordinates of the upper left corner of the target candidate box, $n$ is the number of pixels in subarea bin $(i, j)$, and $\nabla$ represents all the learned parameters of the network. The model calculates the average of pooled response output $r_{c}(i, j \mid \nabla)$ for $k \times k$ subregions and uses the softmax regression classification method to obtain the probability that it belongs to each category.

R-FCN integrates the target's position information into ROI pooling by position-sensitive score map, which solves the problem that the ROI pooling of Faster-RCNN network has no translation invariance. Thus, this model improves the accuracy of target detection and classification so that the operating efficiency of the model is significantly superior. However, it is evident that the RFCN model still cannot detect the specific location of the target and lacks the robustness to the scene of power components with many interfering objects.

\section{Recognition of power components based on Mask LSTM-CNN}

Although the Faster-RCNN and R-FCN methods improve the processing speed and accuracy of part identification models, they cannot refine the specific contours of power components so that live working robots cannot accurately identify components' orientations through such methods.

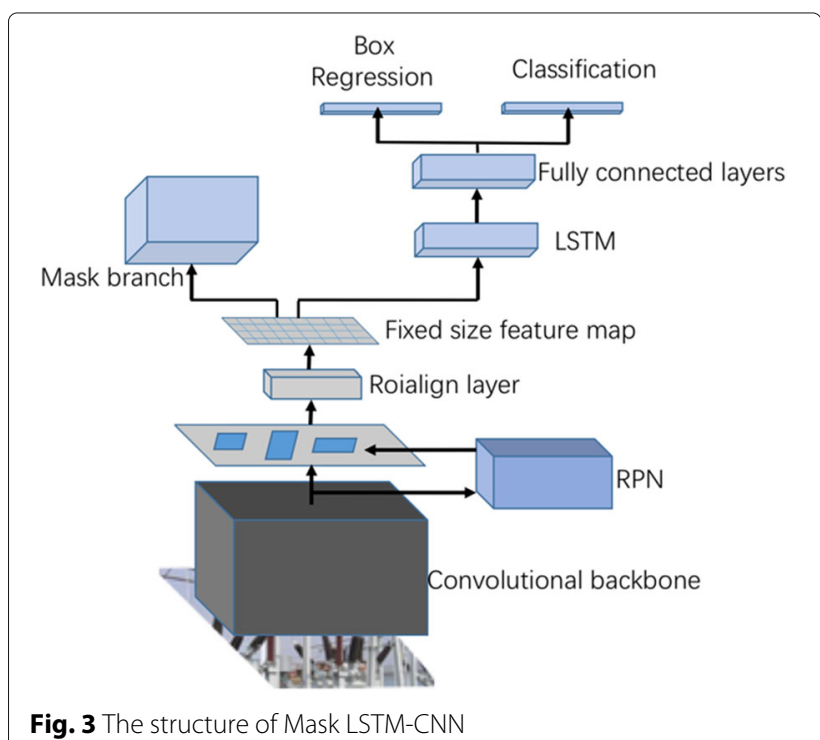




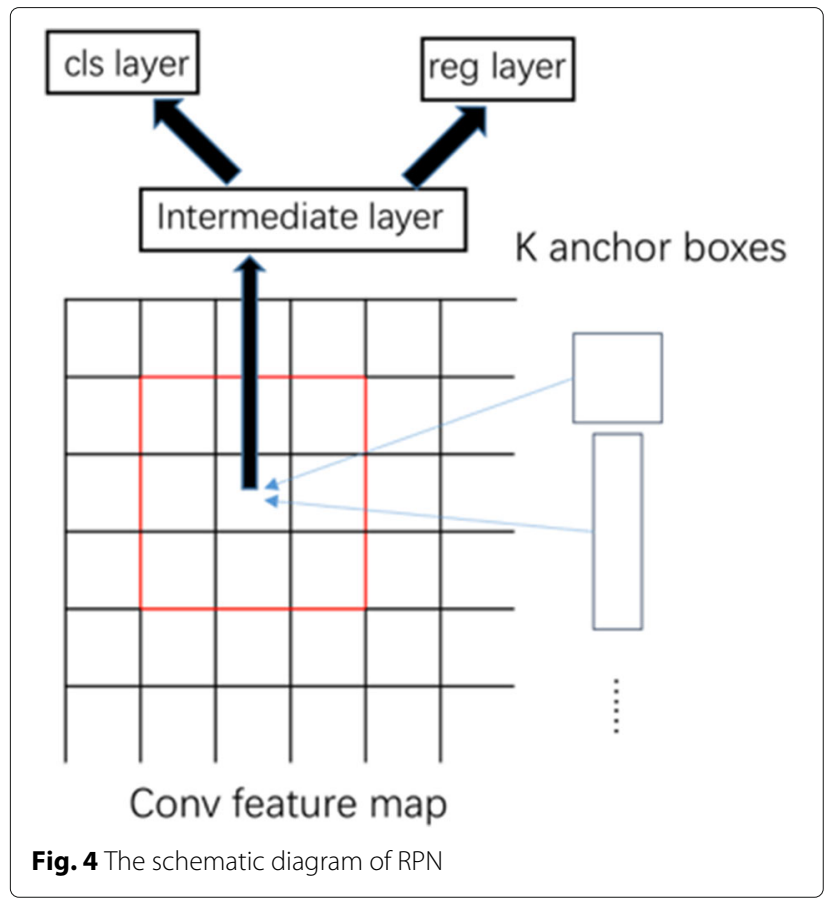

Moreover, the recognition rate of above two methods will obtain an inferior performance and cannot meet the complex industrial environment if power components suffer some. In this section, we combine Mask-RCNN to construct an efficient Mask LSTM-CNN model to sufficiently reduce the influence of obstructions on the recognition of targets.

\subsection{Neural network model for power component identification}

Proposed Mask LSTM-CNN model consists of four parts: pre-training CNN model, RPN network, RoI-Align layer, and detection network layer and Mask layer. The specific structure of the model is shown in Fig. 3. The model uses LSTM to correlate ROI information before the target is identified to reduce the effect of obstacles on the power component. The model improves the accuracy of power component recognition by learning the dependencies between regions.

(1) Pre-training CNN model

Inspired by the existing CNN model, we use ResNet (a further comparison during the experiment) to pretrain the data in the coco2017 image classification task. The data collected from the collected power component inspection data is used to improve and eventually build a complete CNN model. The CNN model is the basis of the proposed method, and it provides the feature map required for subsequent RPN networks and detection networks [24]. The feature map contains features from the deep convolution of the input image, and Euclidean distances between features of objects are proportional to the differences between those objects. That is to say that the feature map can differentiate objects well [25].

(2) RPN network

The power component image generates a multi-channel feature map through the previous CNN network. The RPN network applies a sliding window to these feature maps and uses the anchor mechanism to determine and classify the target region of the feature map. Finally, the back-propagation algorithm is used to tune the regional proposal network.

A plurality of convolution kernels in the output layer is used to perform a convolution operation, and then, a three-dimensional tensor is obtained. The tensor is used as the input of two independent convolution layers to convert the information in the feature map into the position information of the candidate region and the probability information of the context. As shown in Fig. 4, the red area in the figure is the search area. In the picture, only part of the search target box is drawn.

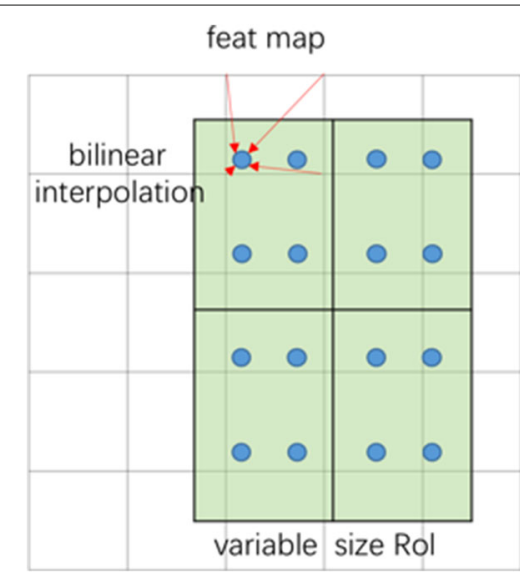

\section{Fixed dimensional Rol output}

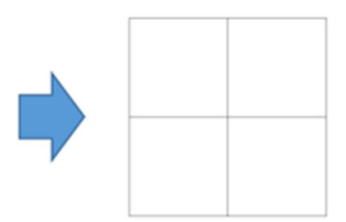

Fig. 5 The schematic diagram of bilinear interpolation 


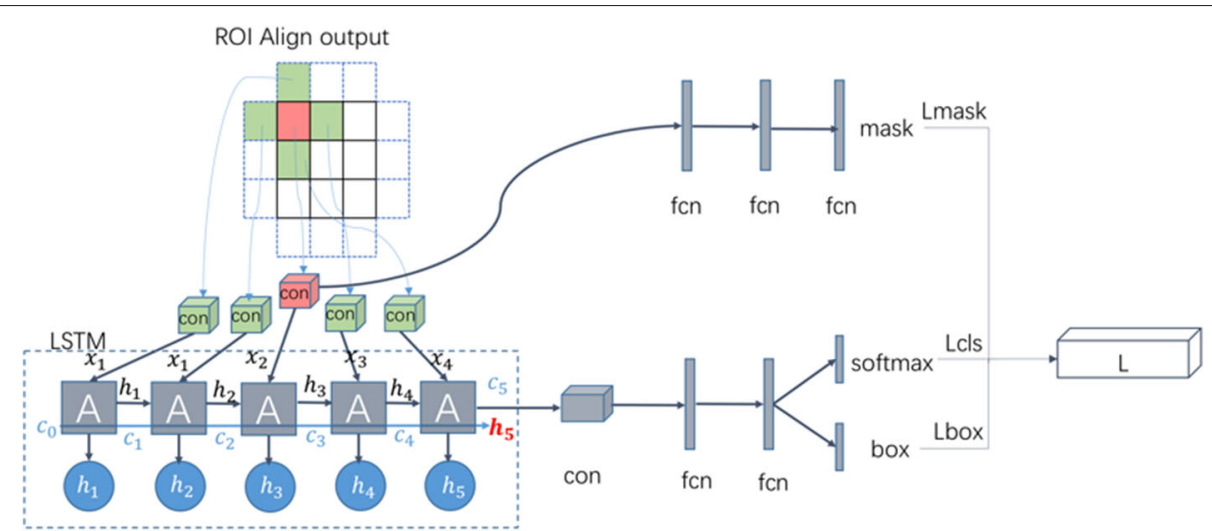

Fig. 6 The structure of detection and Mask layer

RPN uses nine search boxes to search for an area with aspect ratios of 1:1, 1:2, and 2:1. The RPN network can get approximate 20,000 search boxes from an original input image. In practical applications, some search boxes beyond the border of the image are removed. Meanwhile, NMS (non-maximal suppression) [26] method is used to handle the overlapping of search boxes on the same target. The above strategies can significantly improve the search efficiency of candidate target boxes.

RPN completes the search of candidate areas on the output layer of the rudimentary convolutional network and provides candidate areas for the subsequent target detection network, which improves the efficiency of the entire model.

\section{(3) RoI-Align layer}

RoI-Align optimizes the problem of pixel bias and uses bilinear interpolation to obtain the image values at the pixels whose coordinates are floating-point numbers. Finally, the entire feature aggregation process is integrated into continuous operation. ROI-Align layer traverses each candidate areas and keeps floating-point boundaries unquantified. Thexn, it divides the candidate area into $k \times k$ units and holds the boundaries of each unit unquantified. Inside each unit, the fixed four coordinate positions are calculated by bilinear interpolation. The interpolation method calculates the values of these four locations and then performs the maximum pooling operation. The specific process is shown in Fig. 5.

In the back-propagation of the RoI-Align layer, $x_{i} \times(r, j)$ is the coordinate position of a floating point (sample point calculated during forwarding propagation). In the feature map before pooling,each point within the window that has size two by two and centers at $x_{i} \times(r, j)$ should receive the gradient w.r.t the corresponding point $y_{r j}$ gradient, the back-propagation formula of the RoI-Align layer is as follows:

$$
\frac{\partial L}{\partial x_{i}}=\sum_{r} \sum_{j}[d(i, i \times(r, j))<1](1-\Delta k)(1-\Delta w) \frac{\partial l}{\partial y_{r j}}
$$

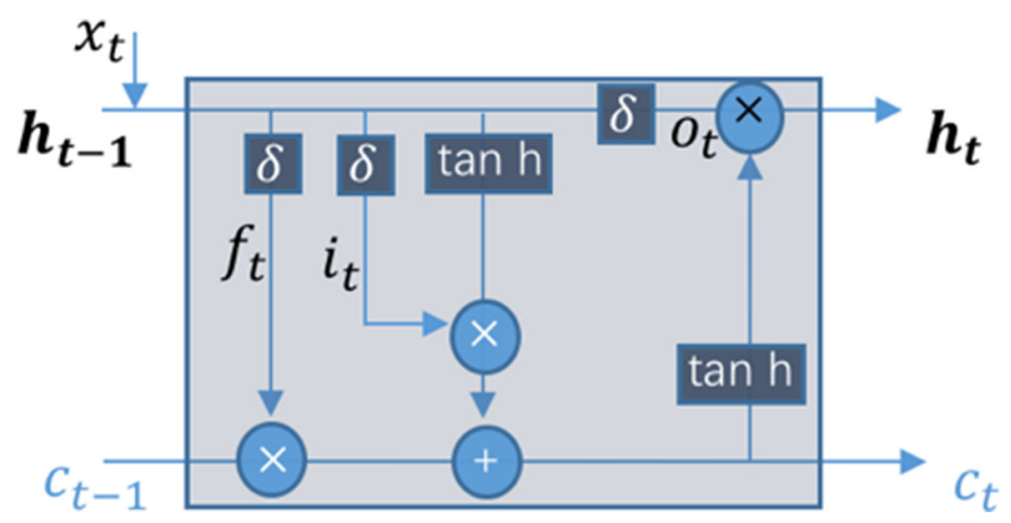

Fig. 7 The structure of hidden layer $A$ 


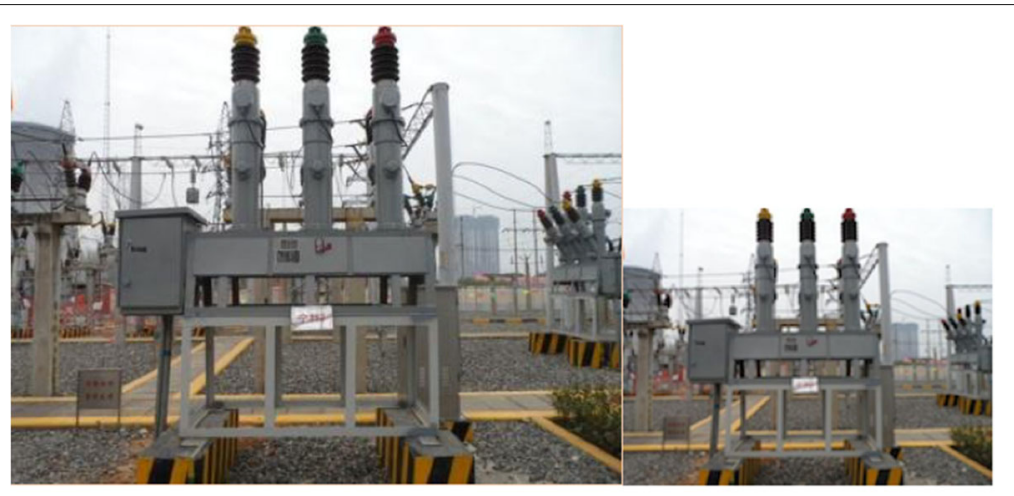

(a)

(b)

Fig. 8 Illustrations in the sample: a "original image" and b "training sample"

where $d$ represents the distance between two points, and $\Delta k$ and $\Delta w$ describe the difference between $x_{i}$, the longitudinal coordinate, and $x_{i} *(r, j)$, the transverse coordinates. Here, the bilinear interpolation coefficient is multiplied by the original gradient.

The RoI-Align layer solves the problem of RoI misalignment between the feature map and the original image, and it obtains better measurement results through more rigorous positioning metrics, which relatively improves the accuracy of the mask.

(4) Detect network layer and Mask layer

The result of the regional proposal generated according to step (3) is the input of the detection network and the Mask layer, wherein the detection network is composed of a classification network and a regional location network. The specific structure is shown in Fig. 6.

The detection network uses the convolutional network to pre-convolve each ROI and its top, bottom, left, and right regions to extract the high-dimensional feature vector as the input to lstm [27][28]. The connection status of the memory unit and various doors are shown in the blue area in Fig. 6 . In the figure, $x_{t}$ denotes the input of different regions, and $h_{t}$ denotes the output of $t$ region. The sigmoid function transforms the input information by multiplying point by point. Forget gate determines whether to save the previous area from the stored state- $h_{t}$. The input gate determines the information that needs to be updated. The entire unit updates the storage status through forget and input gates $c_{t}$. The output gate [29] determines whether to store information in the memory for output. Through five inputs $x_{t}$ and each hidden output $h_{t}$, the final output is $h_{5}$. The concrete structure of hidden layer A is shown in Fig. 7. When the $t$ th block region of the sequence enters the network, the input of the LSTM hidden layer includes the current input $x_{t}$ of the network, the hidden layer output vector $x_{t}-1$ at the previous time, and the hidden layer state $c_{t}-1$. The task of the hidden layer is to calculate and output the vector $h_{t}$ and update the state to obtain $c_{t}$. For this hidden layer, the oblivion gate $f_{t}$, the input gate, and the output $o_{t}$ gate are added. Oblivion gate $f_{t}$ determines which information

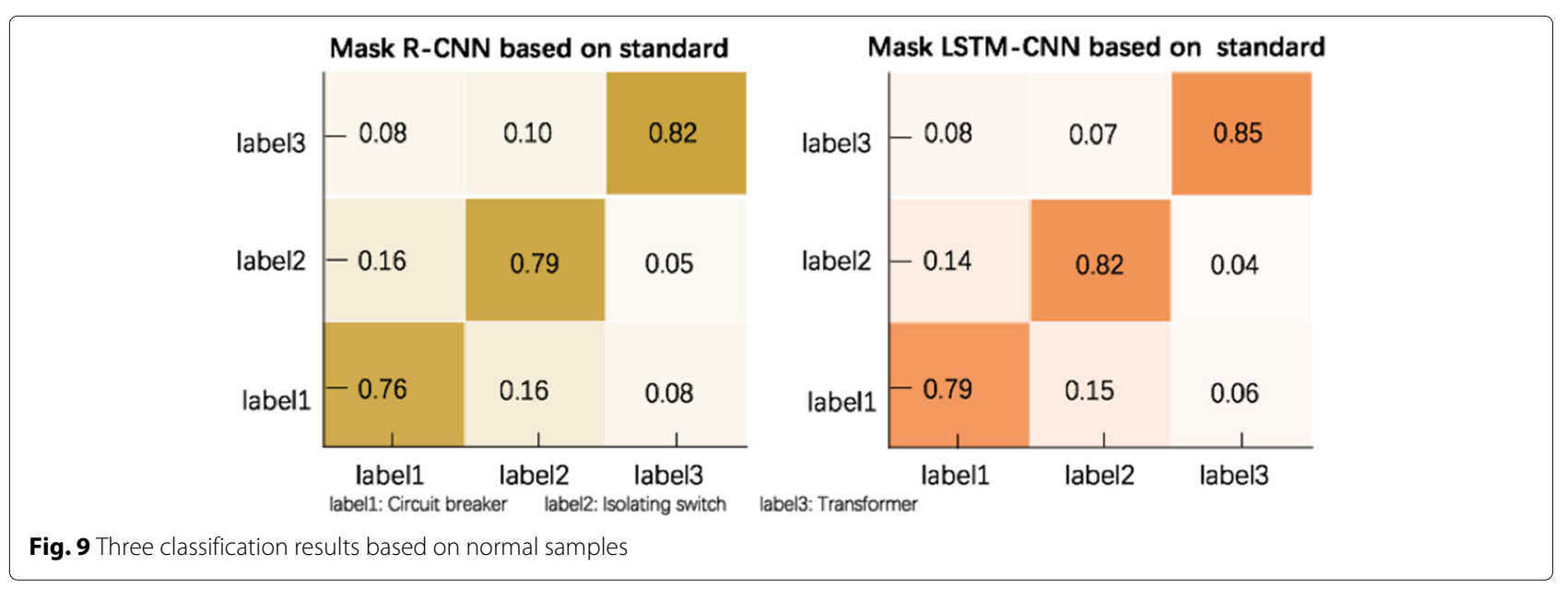



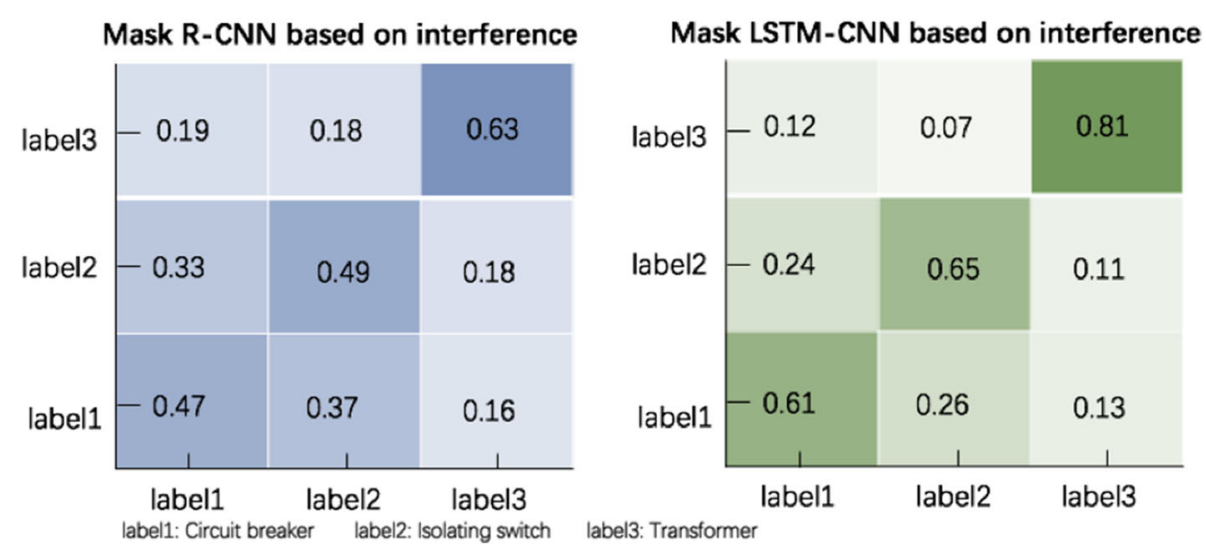

Fig. 10 Three classification results based on disturbed samples

in state $c_{t}$ is discarded. Input gate $i_{t}$ determines which of the updated information from $x_{t}$ and $h_{t}-1$ can be used for status $c_{t}$ updates. After oblivion gate and the output gate, state $c_{t}$ update is completed. The purpose of adding the hidden layer state in the LSTM is to make it affect the output $h_{t}$ of the hidden layer, so the output gate $o_{t}$ is used to determine how the state $c_{t}$ affects the calculation of the $h_{t}$.

The sigmoid activation function is shown in $\delta$ in Fig. 7 . The calculation expressions of the three new additions, the hidden layer output $h_{t}$, and status update $c_{t}$ are calculated as follows:

$$
\begin{gathered}
f_{t}=\delta\left(W_{f} \cdot\left[x_{t}, h_{t-1}\right]+b_{f}\right) \\
i_{t}=\delta\left(W_{i} \cdot\left[x_{t}, h_{t-1}\right]+b_{i}\right) \\
o_{t}=\delta\left(W_{o} \cdot\left[x_{t}, h_{t-1}\right]+b_{o}\right) \\
c_{t}=\tanh \left(W_{c} \cdot\left[x_{t}, h_{t-1}\right]+b_{c}\right)+f_{t} \cdot c_{t-1} \\
h_{t}=o_{t} \cdot \tanh \left(c_{t}\right)
\end{gathered}
$$

The last output connects two layers of full-connected output $k+1$-dimensional array $p$ and $4 \times k$-dimensional array $t$, and array $p$ represents the probability of belonging to class $k$ and background. Output a discrete probability distribution for each RoI (Region of Interesting):

$$
p=\left(p_{0}, p_{1} \cdots, p_{k}\right)
$$

$p$ is computed using softmax from the $k+1$ full connection layer. The array $t$ represents the parameters that should be pan-scaled when belonging to the $k$-type respectively:

$$
t^{k}=\left(t_{x}^{k}, t_{y}^{k}, t_{w}^{k}, t_{h}^{k}\right)
$$

$k$ denotes the index of the category, $t_{x}^{k}$ and $t_{y}^{k}$ are the translations invariant w.r.t the scale of the object proposal, $t_{w}^{k}$ and $t_{h}^{k}$ are the height and width of the object relative to the object proposal in space. The probability corresponding to the real classification $u$ determines the value of loss function $L_{\mathrm{cls}}$ of the classification layer:

$$
L_{\mathrm{cls}}(p, u)=-\log p_{u}
$$

The loss function $L_{\mathrm{box}}$ of box frame detection is obtained by comparing the difference between the prediction panning scaling parameter $t^{u}$ and the real panning scaling

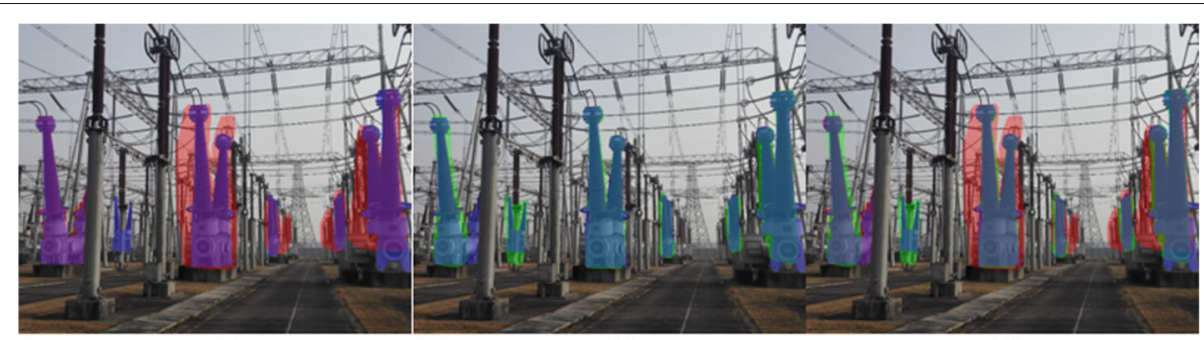

(a)

(b)

(c)

Fig. 11 Proposed model and Mask R-CNN recognition results: (a or red) "Mask R-CNN," (b or green) "Mask LSTM-CNN," c "comparative results," and (blue) "original object" 
parameter $v$, which corresponds to the actual classification. The specific formula is as follows:

$$
\begin{aligned}
v & =\left(v_{x}, v_{y}, v_{w}, v_{h}\right) \\
L_{\text {box }}\left(t^{u}, v\right) & =\sum_{i=1}^{4} \operatorname{smooth}_{L_{1}}\left(t_{i}^{u}-v_{i}\right)
\end{aligned}
$$

Among them, $\operatorname{smooth}_{L_{1}}$ loss function:

$$
\operatorname{smooth}_{L_{1}}(X)=\left\{\begin{array}{l}
0.5 x^{2}(i f|x|<1) \\
|x|-0.5 \text { (otherwise) }
\end{array}\right.
$$

The last layer of the full convolutional layer is predicted from the probability that the candidate region box belongs to each category, the score, and the more appropriate location of the target object's outer frame, which uses four parameters relative to the two region translation and two scaling of the candidate region frame [30].

The Mask layer has an output of $k \times m^{2}$ dimensions for each RoI, $K$ (class number) binary mask with resolution $m \times m$. Therefore, the author uses a per-pixel sigmoid and defines $L_{\text {mask }}$ as the average binary cross-entropy loss. For a RoI belonging to the $k$ th category, $L_{\text {mask }}$ only considers the $k$ th mask (other mask inputs do not contribute to the loss function). Such a definition would allow the algorithm to generate a mask for each category, and there would be no inter-class competition. Mask layer loss function:

$$
L_{\text {mask }}\left(C l s \_k\right)=\operatorname{Sigmoid}\left(C l s \_k\right)
$$

The total loss function can be represented as the sum of the loss functions w.r.t classification error, detection error, and segmentation error.

$$
L=L_{\mathrm{cls}}+L_{\mathrm{box}}+L_{\text {mask }}
$$

Finally, the network is fine-tuned using the backpropagation algorithmthrough pre-marked information [31].

\subsection{Detection and identification process}

As can be seen from the above process, the two networks can eventually share the same characteristic information, which improves the information utilization rate. The RoIAlign layer reduces the loss of spatial symmetry and correlating the knowledge of the upper and lower regions of the ROI enhances the robustness of the model. The Mask layer can achieve pixel-level segmentation.

The process of detection and identification is as follows:

Step 1: A series of convolution operations are performed on the entire image to obtain a feature map.

Step 2: Generate a large number of candidate areas on the feature map by the regional proposal network.

Step 3: Non-maximum suppression of candidate region boxes, retaining the first few boxes with higher scores.

Step 4: Take out the feature in the candidate region frame on the feature map to form a high-dimensional feature vector. Calculate category scores from the detection
Table 1 Comparison of two models mAP, recall, and mEAO

\begin{tabular}{llll}
\hline Model & mAP & mRecall & mEAO \\
\hline Mask R-CNN normal & 0.793 & 0.82 & 0.83 \\
Proposed model normal & 0.833 & 0.85 & 0.91 \\
Mask R-CNN disturbed & 0.532 & 0.67 & 0.64 \\
Proposed model disturbed & 0.694 & 0.78 & 0.79 \\
\hline
\end{tabular}

network and predict more appropriate target peripheral frame positions.

Step 5: The corresponding binary mask is predicted for each feature map according to the classification in (4).

The method shows through experiments that under the identification of power components with obstructions, solved the problem when the recognition rate is low.

\section{Results and discussion}

In this section, we validate the proposed scheme by some images from a real power station. Live working robot captures images with high resolution, including rapid zooming of the target size. The angle of the captured image is diverse and random. Three types of power components are considered: transformers, isolation switches, and circuit breakers.

\subsection{Training sample processing}

The dataset comes from the substation inspection image. The original image size is $1200 \times 900$ (Fig. 8)a, and we intercept the square block image with the target as the main body and uniformly reduce it to $800 \times 600$ (Fig. 8)b as a training sample.

\subsection{Training sets and test sets}

In this test, for each type of component of transformers, isolation switches, and circuit breakers, 1200 training samples were used. A total of 3600 samples constitute a training set; 400 test images of each type and a total of 1200 images constitute a test set. The outer box is marked for the power components in each picture in the training set. For the test set, all the electric components appearing in each picture are marked.

Table 2 Comparison of models mAP based on different basic CNN

\begin{tabular}{llll}
\hline & Faster-RCNN & R-FCN & Proposed model \\
\hline VGG-19 & 0.763 & 0.791 & 0.813 \\
ResNet-50 & 0.781 & 0.797 & 0.831 \\
ResNet-101 & 0.789 & 0.817 & 0.846 \\
ResNeXt-50 & 0.791 & 0.821 & 0.845 \\
ResNeXt-101 & 0.821 & 0.839 & 0.869 \\
\hline
\end{tabular}


Table 3 The comparison of the recall rate of three models based on ResNeXt-101

\begin{tabular}{llll}
\hline & Faster-RCNN & R-FCN & Proposed model \\
\hline mRecall & 0.74 & 0.84 & 0.85 \\
mEAO & 0.83 & 0.87 & 0.92 \\
\hline
\end{tabular}

During the test, it is considered as an auspicious recognition when the overlapped area of the identified outer frame and marked outer frame reaches more than $80 \%$ of the marked outer frame. In this experiment, average precision, recall rate, and effective area occupancy rate are used to judge the accuracy of identification. Among them, the AP (average precision) is as follows:

$$
\mathrm{AP}=\frac{n_{c P}}{n_{c A}}
$$

where $n_{C P}$ indicates the correct number of outer frames for the target category and $n_{c A}$ indicates the number of outer frames marked. The recall rate is as follows:

$$
\text { Recall }=\frac{n_{b P}}{n_{b A}}
$$

$n_{b P}$ is the number of the outer frame that the target category correctly marks, and $n_{b A}$ is the number of all standard outer frames. EAO (effective area occupancy):

$$
\mathrm{EAO}=\frac{m_{P} \& \& m_{A}}{m_{P}}
$$

where $m_{P}$ is the area predicted by the model and $m_{A}$ is the actual area of the target area. Since there are only three types of categories identified in this experiment, mAP (mean average precision), mRecall (mean recall), and mEAO (mean effective area occupancy) of each type of power component are separately counted.

\subsection{Experimental results}

In this section, we use the same rudimentary convolutional network and performance parameters to compare the performance of Mask R-CNN and Mask LSTM-CNN.
Table 4 The time required for three models (based on ResNeXt-10) to deal with each picture

\begin{tabular}{llll}
\hline & Faster-RCNN & R-FCN & Proposed model \\
\hline Time & $300 \mathrm{~ms}$ & $180 \mathrm{~ms}$ & $800 \mathrm{~ms}$ \\
\hline
\end{tabular}

The average precision and recall rate of the two models were tested with 1200 normal samples, as shown in Fig. 9. As can be seen in this figure, the proposed method is slightly higher than Mask R-CNN in classification accuracy of circuit breakers, isolation switches, and transformers. To further test the improved advantages of the proposed method, we prepare a particular sample set including 600 power components with shielding, which are shown in Fig. 10. We can see that Mask LSTM-CNN has a clear advantage over Mask R-CNN in these samples. Figure 11 shows the case that there are many obstructers in images. The blue marker is the actual segmentation result of the sample, the red marker is the segmentation result of the Mask $\mathrm{R}-\mathrm{CNN}$ model, and the green marker is the segmentation result of the Mask LSTM$\mathrm{CNN}$ model. The figure demonstrates that the accuracy of Mask LSTM-CNN segmentation is better than that of Mask R-CNN, and Mask R-CNN identifies more interference backgrounds as part of the target. There are three possible reasons for this exciting phenomenon. The method proposed in this paper incorporates a long-term and short-term memory network before a fully connected decision layer. The method saves the picture information of the upper and lower areas through the intermediate state. The proposed method uses the intermediate state as the input to influence the judgment of the next area. In this way, the proposed model enhances the basis for the model to judge the regional information, and it effectively solves the problem that the model has reduced ability to identify interference factors due to the disappearance of gradients during the training of the model.

Based on the two kinds of samples, further experiments are performed to calculate the mAP, mRecall, and mEAO of the two models under different samples. The experimental statistics are in Table 1. In this table, the recognition effect of Mask LSTM-RNN model on normal

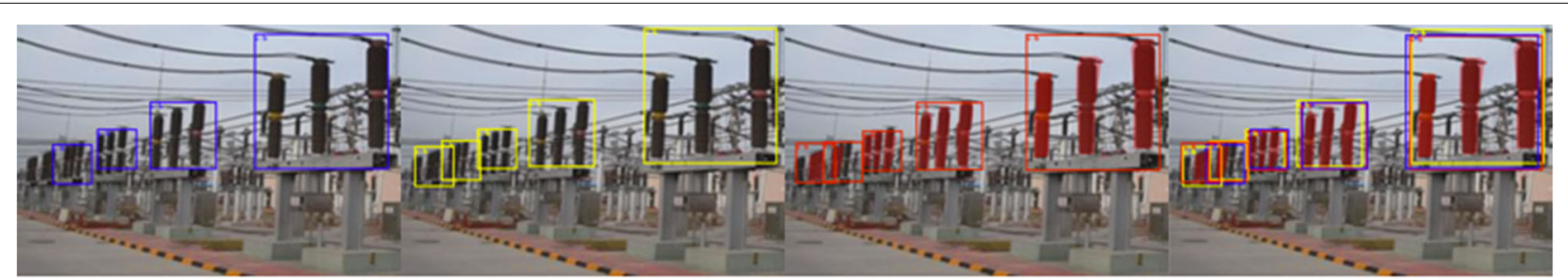

(a) (b) (d)

Fig. 12 The recognition effect of three models on the circuit breaker: (a or blue) Faster-RCNN, (b or yellow) R-FCN, (c or red) Mask LSTM-CNN, and d comparative results 


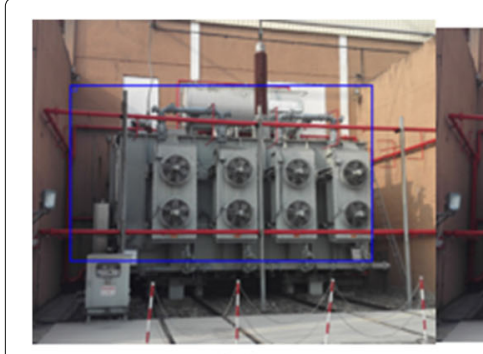

(a)

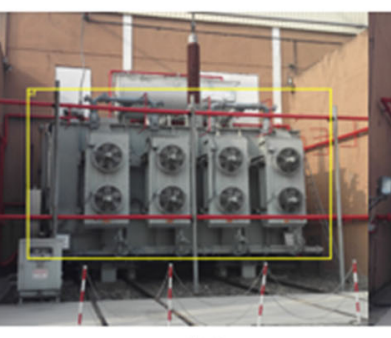

(b)

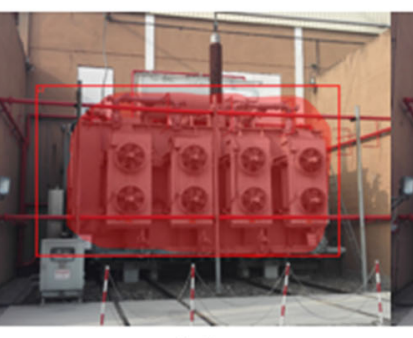

(c)

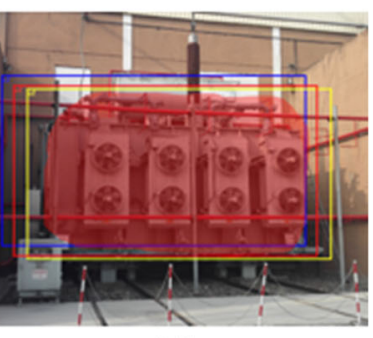

(d)

Fig. 13 The recognition effect of three models on transformer: (a or blue) Faster-RCNN, (b or yellow) R-FCN, (c or red) Mask LSTM-CNN, and d comparative results

samples is better than that of Mask R-CNN model. The recognition accuracy of is evidently superior on samples with obstructions. The mAP surpasses Mask R-CNN with an improvement of $16 \%$, mRecall exceeds with a gain of $11 \%$, and mEAO surpasses Mask R-CNN with an increase of $15 \%$.

The power of the site is usually complicated because there are more obstructions before the power component target. Mask LSTM-CNN demonstrates a more significant improvement in this task. The reason is that Mask LSTMCNN associate ROI information with LSTM before box identification. Through the image information of the nearby areas, it helps to judge the existence of obstacles, strengthens the judgment basis of the neural network, and further improves the accuracy of the classification. And the judgment of the Mask layer depends on the classification result. The result of the classification determines the type of mask that the target generates, so the accuracy of the classification layer directly relates to the accuracy of the Mask.

In addition, we use different basic convolutional frameworks such as VGG, ResNet, and ResNet as the basic network of RCNN and compare the effects of different basic convolutional frameworks on the accuracy of FasterRCNN, R-FCN, and Mask LSTM-CNN models with the same performance parameters. The classification results and the regional selection of mAP (mean average precision) are shown in Tables 2 and 3.

From Table 2, we conclude that the mAP of the model's underlying network when using ResNet is higher than that of VGG. When the model uses ResNet as the underlying network, its map is highest. When the model uses ResNet-101 as the underlying convolutional network, its $\mathrm{mAP}$ is as high as $87 \%$. The reason is that ResNet proposes a residual structure compared to VGG. Through reformulation, ResNet decomposes a problem into multiple scales and direct residual issues, which can be used to optimize the training effect. ResNet retains ResNet's stacking blocks. ResNet splits a single path, simplifying the model structure and improving computational efficiency.
From the side comparison in Table 2, the LSTM-CNN of Mask LSTM-CNN has significantly enhanced mAP on Faster-RCNN and R-FCN in three basic convolutions. Because the method proposed in this paper contains the mask layer structure, it enables identification of the model at the pixel level, which ultimately leads to a higher recognition rate.

From Table 3, the mRecall of Mask-RCNN and R-FCN is almost equal, which is better than Faster-RCNN. ResNet101-based Mask LSTM-CNN mEAO is significantly better than the other two models.

We further compare the time required for the three algorithms to process each image based on the same basic convolutional network. Results in Table 4 show the R-FCN model has the fastest processing speed. Mask LSTM-CNN is significantly slower than the other two algorithms, but it is also within the acceptable range.

Mask LSTM-CNN is better than Faster-RCNN and R$\mathrm{FCN}$ in both mAP and recall rate. It takes more time to process each picture than the other two models. However,

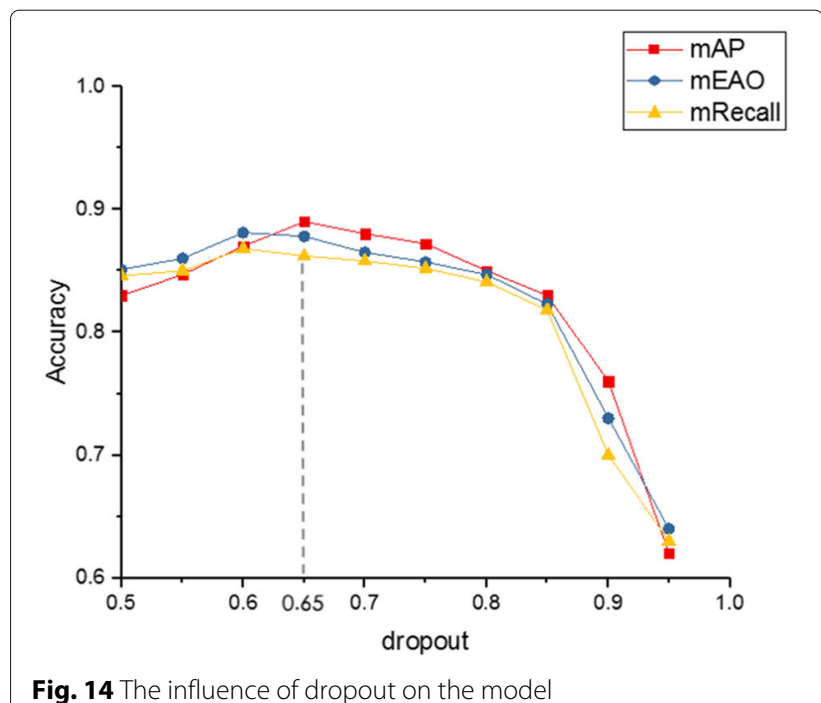




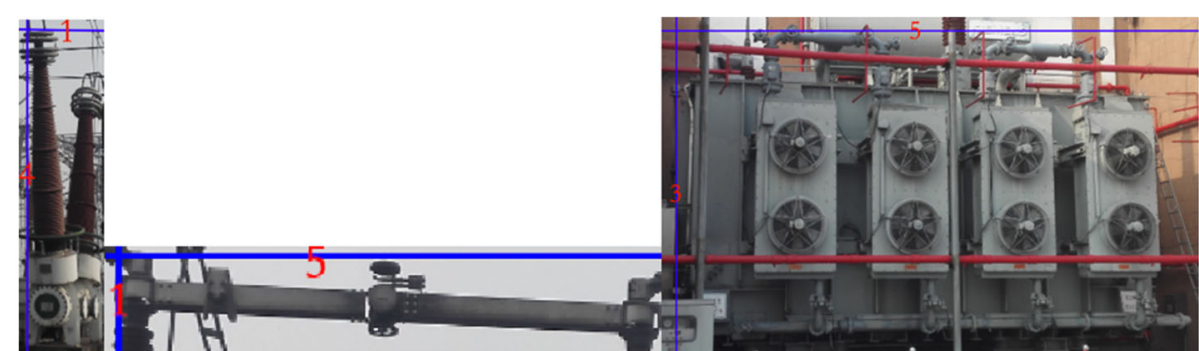

(a)

(b)

(c)

Fig. 15 The approximate size of the three targets: a circuit breaker, $\mathbf{b}$ isolating switch, and $\mathbf{c}$ transformer

Mask LSTM-CNN can refine the detailed profile of components. It is more conducive to the live working robot to determine the position of the various parts of the parts and the details of the accessories, and it has a better adaptation to the target shielding. These advantages are due to the alignment between the extracted features and the input of the ROI-Align layer in the Mask-RCNN model. The proposed model uses the matrix instead of vectors to predict each ROI. It reduces the loss of spatial information. The method proposed in this paper associates the features in the nearby ROI before the fully connected layer. This approach enhances the robustness of object recognition with obstacles. It effectively solves the problem of the gradient disappearing during the process of judging the occlusion object information by a model.

From the following experimental results, as shown in Figs. 12 and 13, the recognition effects of the three models can be seen. The red mark represents the Mask LSTM$\mathrm{CNN}$, the yellow mark represents the R-FCN, and the blue mark represents the Faster-RCNN. Among the Faster$\mathrm{RCNN}$ tagging boxes, the correct number of outer boxes in the target category tag is less than the actual number of outer peripheral boxes. The Mask LSTM-CNN and RFCN are relatively accurate, and the Mask LSTM-CNN can refine the specific outline of the part.

According to the previously mentioned advantages of Mask LSTM-CNN, we optimize the performance of the
Mask LSTM-CNN model based on ResNet-101. The Mask LSTM-CNN model involves some parameters, such as dropout ratio and nms (non-maximum suppression), and the number and size of the ratio and anchor. These parameters have a more significant impact on the $\mathrm{mAP}$, and we will discuss them as follows.

Due to the massive time consumption of network training, we only tried 10 different dropouts. As shown in Fig. 14, when the dropout ratio increases from 0.5 to 0.95 , the accuracy gets the maximum value at approximate 0.65 . At present, dropout often depends on experience. We traverse according to the specific experimental environment and obtain the relatively optimal value. According to the above experimental results, we set dropout as 0.65 and change the number of anchors. The number of commonly used anchors is set to 9, which belong to three categories and have three aspect ratios $(1: 1,1: 2,2: 1)$. Since the scene identified this time is a power accessory, the recognition object has a fixed characteristic. According to the size ratio of the power accessories, the anchor is further optimized in this paper.

In experimental results in Fig. 15, the size of the isolation switch in the area of about 5:1 is more appropriate, while 1:4 for the circuit breaker and $5: 3$ for the transformer.

Based on these three ratios, three new dimensions are scaled. This article adds nine new anchors. These

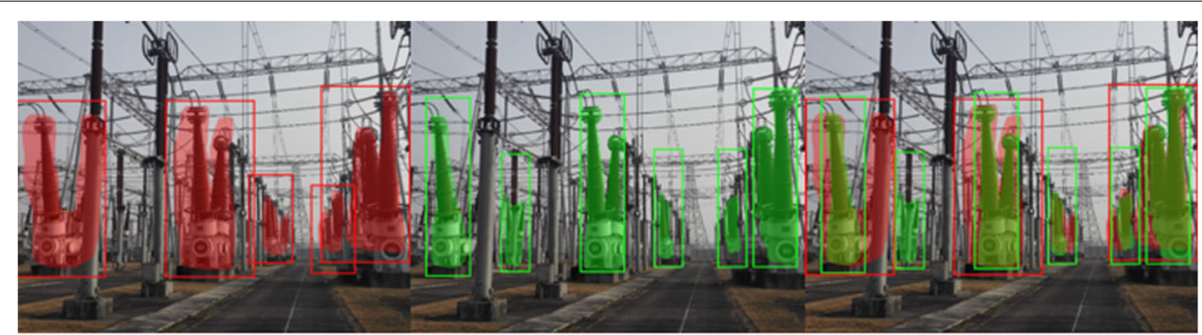

(a)

(b)

(c)

Fig. 16 Comparison of the recognition effect of the model to the circuit breaker: (a or red) "unimproved model," (b or green) "improved model," and c "comparative results" 


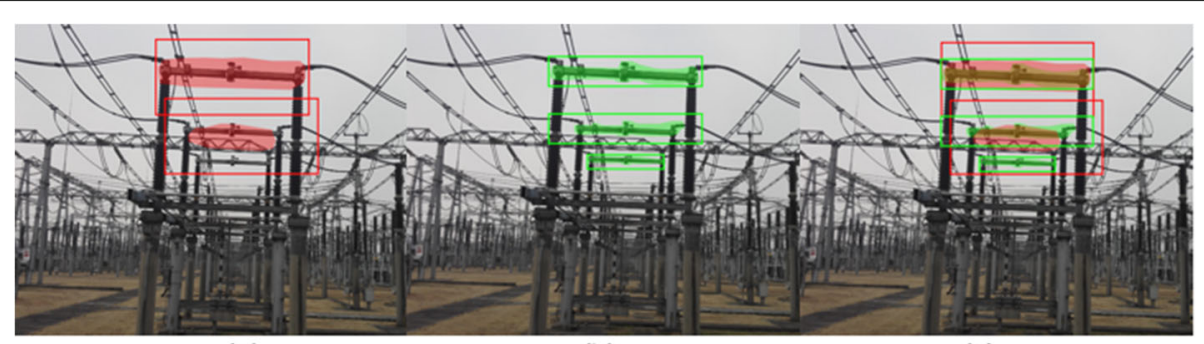

(a)

(b)

(c)

Fig. 17 Comparison of the recognition effect of the model to the isolation switch: (a or red) unimproved model, (b or green) improved model, and c comparative results

nine anchors are used to obtain the various parts of the power device, making us more comprehensive and accurate. Further experiments were conducted to compare with the original model. The experimental results are in Figs. 16 and 17. The red mark indicates that the recognition effect of the model is not improved without nine anchors, and the green mark indicates the recognition effect of the improved model with nine anchors. The Mask LSTM-CNN model is more accurate in the area selection of power components, and the contour recognition of components is more accurate. In regions with high density of power components, there is a significant improvement in recall rate of recognition. According to Table 5 (box-map is the average accuracy of the area recognition, and Mask LSTM-CNN means the average accuracy of the mask), the improved power component recognition model has a noticeable improvement in area selection, contour subdivision, and recall rate.

The model uses a dropout of 0.67 and an anchor type of 18 . It changes the image crossover ratio of the nms to IoU and tests its impact on mAP. The result is in Fig. 18. According to Fig. 18, as the IoU of nms decreases, mAP also gradually decreases. The reason is that the smaller the IoU is, the fewer the candidate areas remain after $\mathrm{nms}$, which leads to a decrease in the accuracy of the detection result. A higher IoU cannot achieve the goal of eliminating the redundancy box by increasing the efficiency of the nms. Therefore, point A in Fig. 18 corresponds to IoU of 0.86 . After point $\mathrm{A}$, the model's accuracy growth has been slow. Thus, the image of the $\mathrm{nms}$ IoU ratio is 0.86 .

\subsection{Discussion}

We have presented corresponding experimental results in above section. The proposed method is compared with several state-of-the-arts, e.g., original Mask R-CNN

Table 5 The influence of the number of A on the model

\begin{tabular}{llll}
\hline Anchor-number & Box-map & Mask-map & Recall \\
\hline 9 & 0.886 & 0.834 & 0.874 \\
18 & 0.893 & 0.867 & 0.943 \\
\hline
\end{tabular}

method and Faster-RCNN, R-FCN, and other methods. Objectively, since we integrate context features in the classification and regression layers, the proposed method obtains better parameter values, such as mAP, mRecall, and mEAO. Tables 1, 2, and 3 show that Mask LSTM$\mathrm{CNN}$ is superior to Mask R-CNN, faster-RCNN, and $\mathrm{R}-\mathrm{FCN}$, and the average accuracy is up to $93 \%$. This demonstrates that our proposed method is more effective. Subjectively speaking, the method proposed in this paper is better than Mask R-CNN, Faster-RCNN, and RFCN in identifying power components. Especially when the power components are blocked, the method proposed in this paper is greatly improved in the recognition rate.

Actually, Mask LSTM-CNN can associate ROI information with LSTM before target classification. A series of convolution operations are performed on the entire image to obtain a feature map: First, generation of a large number of candidate areas on the feature map by the regional proposal network. Next, non-maximum suppression of candidate region boxes, retaining the rst few boxes with higher scores. Then, taking out the feature in the candidate region frame on the feature map to form a highdimensional feature vector. Last, calculation of category

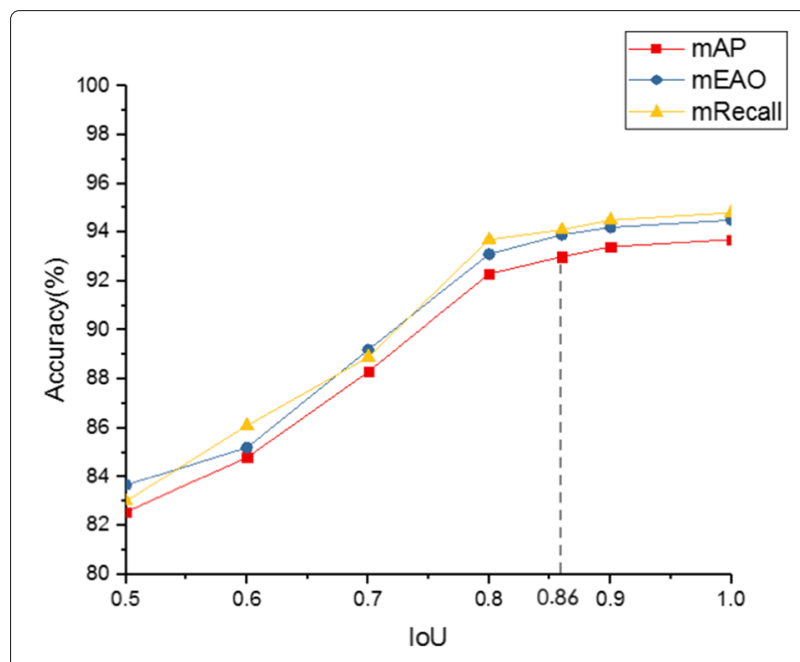

Fig. 18 The influence of lou on the model 
scores from the detection network and prediction of more appropriate target peripheral frame positions. Finally, the classification accuracy is improved significantly. Since the judgment of the Mask layer depends on the classification result, which determines the type of mask that the target generates, the accuracy of the classification layer directly relates to the accuracy of the Mask.

This paper provides the research theory for the image recognition and target positioning of the charged detection robot. Even with complicated working conditions on the scene, the identification accuracy for power components is still improved greatly. Moreover, we design a longterm and short-term memory network to further improve the recurrence of operations and reduce the recognition speed. In the further work, we hope to lower the computational efficiency of the model. A distributed deep neural network may help us to solve this problem. This issue is left as our future work.

\section{Conclusions}

Based on the analysis of the current and more advanced methods for target detection and identification, this paper verifies the accuracy and efficiency of the recognition of power small parts using Mask LSTM-CNN algorithm. The influence of different parameters on the detection results of Mask LSTM-CNN was analyzed. After combining the features of power components to further optimization of the model, experiments show that Mask LSTM$\mathrm{CNN}$ model can accurately detect, locate the power components in real time, and provide a good foundation for automatic maintenance of components in live working robots.

Finally, we point out that a more extensive sample library that can further improve the identification performance. In this sense, there may be room for further improvement. Also, a more elaborate identification category, including the types of fault images for various power components might also be helpful to improve the performance. In addition, it is also possible to apply image detection and recognition in other fields. The above three issues are left as our future work.

\section{Abbreviations \\ AP: Average precision; EAO: Effective area occupancy; Faster-RCNN: Faster region-based convolutional neural network; HOG: Histogram of oriented gradients; LSTM: Long short-term memory; mAP: Mean average precision; mEAO: Mean effective area occupancy; mRecall: Mean recall; NMS: \\ Non-maximal suppression; R-FCN: Region-based fully convolutional networks; RPN: Regional proposal network; SIFT: Scale-invariant feature transform}

\section{Acknowledgements}

The authors would like to thank the editors and anonymous reviewers for their valuable suggestions and comments.

\section{Funding}

This work was supported by the Natural Science Foundation of China under grant nos. 61672337 and 61472236 and Natural Science Foundation of Shanghai grant no. 16ZR1413100.

\section{Availability of data and materials}

Data would not be shared and available right now. The reason for not sharing the data and materials is that the work submitted for review is not completed. The research is still ongoing, and those data and materials are still required by the author and co-authors for further investigations. We will share the data online after the paper is published. People with any questions are welcome to contact us.

\section{Authors' contributions}

$J L$ designed the proposed algorithm and drafted the manuscript. WS designed and conducted the subjective experiments. FL offered useful suggestions and helped to modify the manuscript. ZL participated in the algorithm design and tested the proposed algorithm. WS conducted the subjective experiment and performed the statistical analysis. All authors read and approved the final manuscript.

\section{Competing interests}

The authors declare that they have no competing interests.

\section{Publisher's Note}

Springer Nature remains neutral with regard to jurisdictional claims in published maps and institutional affiliations.

\section{Author details}

${ }^{1}$ College of Computer Science and Technology, Shanghai University of Electric Power, Shanghai, 200090 People's Republic of China. ${ }^{2}$ Paul G. Allen School of Computer Science and Engineering, University of Washington, Seattle, 98105 USA. ${ }^{3}$ College of Automation Engineering, Shanghai University of Electric Power, Shanghai, 200090 People's Republic of China.

Received: 29 May 2018 Accepted: 17 September 2018

Published online: 07 November 2018

\section{References}

1. J. Y. Park, B. H. Cho, S. H. K. Byun, Development of cleaning robot system for live-line suspension insulator strings. Int. J. Control. Autom. Syst. 7(2), 211-220 (2009)

2. C. Liao, J. Ruan, C. Liu, Helicopter live-line work on 1000-kV UHV transmission lines. IEEE Trans. Power Deliv. 31(3), 982-989 (2016)

3. C. Chen, J. Wang, H. Zhu, Effects of phasor measurement uncertainty on power line outage detection. IEEE J. Sel. Top. Sig. Process. 8(6), 1127-1139 (2014)

4. S. Fu, Y. Zhang, L. Cheng, Z. Liang, Z. Hou, M. Tan, Motion based image deblur using recurrent neural network for power transmission line inspection robot. in Proc. of the JICNN'06 Int. Joint Conf. Neural Netw. 3854-3859 (2006)

5. P. Dehghanian, M. Fotuhi-Firuzabad, S. Bagheri-Shouraki, Critical component identification in reliability centered asset management of power distribution systems via fuzzy AHP. IEEE Syst. J. 6(4), 593-602 (2012)

6. Y. Hou, D. I. Jianming, Application of improved scale invariant feature transform accurate image matching in target positioning of electric power equipment. Proc. Csee. 32(19), 134-139 (2012)

7. K. Bowyer, C. Kranenburg, S. Dougherty, Edge detector evaluation using empirical ROC curves. Proc. IEEE Conf. Comput. Vision and Pattern Recognition. (Elsevier Science Inc, 2001)

8. N. Dalal, B. Triggs, Histograms of oriented gradients for human detection. in Proceedings of the IEEE conference on computer vision and pattern recognition. 886-893 (2005)

9. B. Yangel, D. Vetrov, Image segmentation with a shape prior based on simplified skeleton, Energy Minimization Methods in Computer Vision and Pattern Recognition, St. Petersburg, 247-260 (2011)

10. S. Wei, Q. Hong, M. Hou, Automatic image segmentation based on PCNN with adaptive threshold time constant. Neurocomputing. 74(9), 1485-1491 (2011)

11. S. Ren, K. He, R. Girshick, J. Sun, Faster R-CNN: towards real-time object detection with region proposal networks. in Neural Information Processing Systems (NIPS), 91-99 (2015)

12. J. Dai, Y. Li, K. He, J. Sun, R-fcn: Object detection via region-based fully convolutional networks. arXiv preprint arXiv:1605.06409 (2016) 
13. K. He, G. Gkioxari, P. Dollar, Mask R-CNN. IEEE Int. Conf. Comput. Vis. 2980-2988 (2018). Venice

14. E. J. Kirkland, Bilinear interpolation. Adv. Comput. Electron Microsc 261-263 (2010). Springer, Boston

15. A. Graves, J. Schmidhuber, Framewise phoneme classification with bidirectional LSTM networks. IEEE Int. Joint Conf. Neural Netw. 18(5-6), 602-610 (2005)

16. $\mathrm{G} . \mathrm{Xu}, \mathrm{An}$ adaptive parameter tuning of particle swarm optimization algorithm. Appl. Math. Comput. 219(9), 4560-4569 (2013)

17. S. Ren, K. He, R. Girshick, et al, Faster R-CNN: towards real-time object detection with region proposal networks. IEEE Transactions on Pattern Analysis \& Machine Intelligence. 39(6), 1137-1149 (2017)

18. R. Girshick, Fast R-CNN. IEEE Int. Conf. Comput. Vis., IEEE, 1440-1448 (2015)

19. J. R. R. Uijlings, K. E. A. V. D. Sande, T. Gevers, Selective search for object recognition. Int. J. Comput. Vis. 104(2), 154-171 (2013)

20. J. Liu, T. Ren, Y. Wang, S. H. Zhong, J. Bei, S. Chen, Object proposal on RGB-D images via elastic edge boxes. NEUCOM. 236, 134-146 (2017)

21. L. F. Palafox, C. W. Hamilton, S. P. Scheidt, Automated detection of geological landforms on mars using convolutional neural networks. Comput. Geosci. 101, 48-56 (2017)

22. K. Simonyan, A. Zisserman, Very deep convolutional networks for large-scale image recognition. Int. Conf. Learn. Representations. CoRR, abs/1409.1556 (2014)

23. D. Sarikaya, J. Corso, K. Guru, Detection and localization of robotic tools in robot-assisted surgery videos using deep neural networks for region proposal and detection. IEEE Trans. Med. Imaging. PP(99), 1-1 (2017)

24. J. Koh, M. Suk, S. M. Bhandarkar, A multilayer self-organizing feature map for range image segmentation. Neural Netw. 8(1), 67-86 (1995)

25. B. Sowmya, B. S. Rani, Colour image segmentation using fuzzy clustering techniques and competitive neural network. Appl. Soil Ecol. 11(3), 3170-3178 (2011)

26. N. Dalal, Finding people in images and video. Grenoble Inst. Natl Polytechnique de Grenoble-INPG. (2006)

27. M. Weber, M. Liwicki, D. Stricker, C. Scholzel, S. Uchida, LSTM-based early recognition of motion patterns. in ICPR. IEEE, 3552-3557 (2014)

28. M. F. Stollenga, W. Byeon, M. Liwicki, et al., Parallel multi-dimensional LSTM, with application to fast biomedical volumetric image segmentation. Comput. Sci. (2015)

29. J. Song, S. Tang, J. Xiao, LSTM-in-LSTM for generating long descriptions of images. Comput. Vis. Media. 2(4), 1-10 (2016)

30. M. A. Rafique, W. Pedrycz, M. Jeon, Vehicle license plate detection using region-based convolutional neural networks. Soft. Comput. 3, 1-12 (2017)

31. Y. Hirose, K. Yamashita, S. Hijiya, Back-propagation algorithm which varies the number of hidden units. Int. Joint Conf. Neural Netw. 2, 625 (1991)

\section{Submit your manuscript to a SpringerOpen ${ }^{\circ}$ journal and benefit from:}

- Convenient online submission

- Rigorous peer review

- Open access: articles freely available online

- High visibility within the field

- Retaining the copyright to your article

Submit your next manuscript at $\gg$ springeropen.com 